\title{
Influence of atmospheric electric fields on radio emission from air showers.
}

\author{
Olaf Scholten* \\ University of Groningen, KVI Center for Advanced Radiation Technology, 9747 AA Groningen, \\ The Netherlands \\ and Interuniversity Institute for High-Energy, Vrije Universiteit Brussel, Pleinlaan 2, 1050 \\ Brussels, Belgium \\ E-mail: O. Scholten@rug.nl
}

\section{Gia Trinh}

Department of Physics, School of Education, Can Tho University Campus II, 3/2 Street, Ninh Kieu District, Can Tho City, Vietnam

E-mail: ttngia@ctu.edu.vn

\section{Ute Ebert}

CWI, Centrum Wiskunde \& Informatica, Amsterdam, The Netherlands

TU/e, Eindhoven University of Technology, Eindhoven, The Netherlands

\section{for the LOFAR CR-KSP}

\begin{abstract}
We show that atmospheric electric fields as exist in thunderclouds can strongly affect the radio emission from cosmic air showers. We also show, using data from LOFAR, that from the measured radio footprint of cosmic-ray air showers, i.e. intensity, linear and circular polarization at various distances from the shower core, one can determine the direction and strength of the electric field as function of height along the path of the cosmic ray. This method can be regarded as tomography of thundercloud electric fields using cosmic rays as probes. We will present an analysis of selected events measured during thunderstorm conditions in the period from December 2011 till August 2014. The fields we extract are consistent with the generally accepted charge structure in thunderclouds consisting out of three charge layers.
\end{abstract}

36th International Cosmic Ray Conference -ICRC2019-

July 24th - August 1st, 2019

Madison, WI, U.S.A.

${ }^{*}$ Speaker. 


\section{Introduction}

Extensive air showers (EAS) induce electric currents, a longitudinal current arising from the charge excess in the shower front and a transverse current arising from the action of the Lorentz force due to the magnetic field of Earth. These currents emit radio waves since their strength changes as function of height in the atmosphere because of the changing number of particles in the EAS $[1,2,3]$. In addition, since the showers proceed faster than the propagation velocity of light in air, there is a contribution from the Cherenkov effects [4, 5]. The emitted radio pulse has proven to be very useful for an efficient determination of the properties of the cosmic ray that initiated the EAS $[6,7]$. This determination of the shower properties relies on a good understanding of the emission mechanisms. In Section 2 we discuss an additional mechanism that contributes to radio emission resulting from the currents induced by atmospheric electric fields in charged clouds [8]. Atmospheric electric fields may induce rather different intensity patterns, but their contribution can most easily be recognized from abnormal polarization footprints as shown in Section 3. In turn it is shown in Section 4 that the radio footprint (intensity and polarization) can be used as a tool to study electric fields in clouds.

\section{Radio emission due to atmospheric electric fields}

It is well known that lightning is driven by strong electric fields in large Cumulonimbus clouds. These electric fields result from a separation of charges due to strong convection flows of ice particles in clouds [9]. It is less well known that strong electric fields may also be present in more ordinary clouds.

When an EAS is developing in a region with an atmospheric electric field the electrons and positrons in the shower front experience an electric force in addition to the geomagnetic Lorentz force. Even for moderate electric fields of $10 \mathrm{kV} / \mathrm{m}$ the electric force is larger than the geomagnetic one. In considering the effect of the electric field one should distinguish between the component perpendicular to the shower axis and the one parallel to it [8]. The effect of the perpendicular component is to induce a transverse current along its direction which in general differs from that of the Lorentz force. This will reflect in the direction of the dominant polarization of the radio emission. The parallel component has a very small effect. The reason for this is that the gain/loss of energy due to this component is negligible for the energetic particles in the shower. Only lower energy particles will be affected, but due to a lack of coherence, as these are found at large distances behind the shower front, this will hardly affect the radio emission in the frequency regime above $30 \mathrm{MHz}$ where most of the observations are made. In addition, effects are balanced since a field that accelerates electrons will decelerate positrons. In leading order the total number of charged particles is thus not affected.

An interesting aspect to note is that when the EAS passes through a charge layer in a cloud there is a change in direction of the force acting on the particles in the shower front. As a result the direction of the induced currents changes as function of height. This gives rise to many interesting phenomena, such as constructive and destructive interference of radiation coming from different heights when the currents flip sign. When the currents at different heights are at an angle the received radio pulse will show a strong circular polarization [10, 11, 12]. 


\section{Radio footprints}

To analyze the radio footprint we use the real-valued Stokes parameters, defined as [10]

$$
\begin{aligned}
& I=\frac{1}{n} \sum_{i=0}^{n-1}\left(\left|\varepsilon_{i, \mathbf{v} \times \mathbf{B}}\right|^{2}+\left|\varepsilon_{i, \mathbf{v} \times(\mathbf{v} \times \mathbf{B})}\right|^{2}\right), \\
& Q=\frac{1}{n} \sum_{i=0}^{n-1}\left(\left|\varepsilon_{i, \mathbf{v} \times \mathbf{B}}\right|^{2}-\left|\varepsilon_{i, \mathbf{v} \times(\mathbf{v} \times \mathbf{B})}\right|^{2}\right), \\
& U+i V=\frac{2}{n} \sum_{i=0}^{n-1}\left(\varepsilon_{i, \mathbf{v} \times \mathbf{B}} \varepsilon_{i, \mathbf{v} \times(\mathbf{v} \times \mathbf{B})}^{*}\right),
\end{aligned}
$$

where $\varepsilon_{i}=S_{i}+i \hat{S}_{i}$ is the complex-valued radio signal, where $\hat{S}_{i}$ is sample $i$ of the Hilbert transform of $S$. These Stokes parameters can be evaluated for each antenna. Stokes $I$ corresponds to the intensity of the radio signal, while $Q$ is the intensity difference between the polarized intensity in the $\mathbf{v} \times \mathbf{B}$ direction and the $\mathbf{v} \times(\mathbf{v} \times \mathbf{B})$ direction. For a fair-weather shower one thus expects $Q / I \approx 1$ since the signal is dominated by geomagnetic emission. Stokes $U$ it the difference in intensity between the polarization directions at $45^{\circ}$ and $-45^{\circ}$ with respect to the $\mathbf{v} \times \mathbf{B}$ axis. For a fair weather event a non-zero value is due to the charge-excess contribution and the sign (and magnitude) depends on the azimuthal angle of the antenna with respect to the $\mathbf{v} \times \mathbf{B}$-axis. Stokes $V$ shows the circular polarization. As argued in Ref. [13] the circular polarization in fair-weather showers results from a slight emission-time difference between charge-excess and geomagnetic radiation and sign and magnitude depend on the azimuth angle of the antenna, similar as for $V / I$. An example of the Stokes parameters for a fair-weather shower can be found in Ref. [13].

For events recorded at LOFAR [14] while there were clouds overhead with (apparently) strong electric fields we find completely different footprint signatures. Two examples are given as events A \& B in Fig. 1 and Fig. 2. Both of these events were recorded on August 26, 2012 where event A was measured at 13:52:23 UTC and event B at 14:02:56 UTC within a 10 minute time span. At the time of observation there was lightning activity in the vicinity of the LOFAR core. It should be noted that clear non-fair-weather radio footprints have also been measured while there was no lightning activity within $200 \mathrm{~km}$ from the core within 12 hours before or after the event.

The intensity and circular polarization patterns observed in event A, see red points in Fig. 1, resemble those of a fair weather event however the linear polarization is rather different. Instead of unity we find here $Q / I \approx 0$ which means that the polarization vector is making a $\pm 45^{\circ}$ angle with the $\mathbf{v} \times \mathbf{B}$ direction. Since $U / I \approx-1$ the angle is actually $-45^{\circ}$. The fact that the circular polarization is small, $V / I \approx 0$, implies that the fields in the different layers are mostly oriented along the same direction as there should not be a net rotation angle in the fields. The blue points in Fig. 1 show the results of a CoREAS calculation [15] using the three-layered structure for the atmospheric electric field as specified in Table 1. For the true force acting on the electrons and positrons the geomagnetic force has to be added, resulting is a net force in the $-45^{\circ}$ plane.

The radio-intensity pattern for event $\mathrm{B}$, occurring only 10 minutes later, is given by the red points in Fig. 2. This shows a clear ring-structure in intensity with a diameter of close to $200 \mathrm{~m}$. This is a clear indication of a strong destructive interference between different layers. The strong circular polarization near the core is evidence that the field orientations have a definite twist. From the values of the atmospheric electric field given in Table 1 one can see that this is indeed the case. 

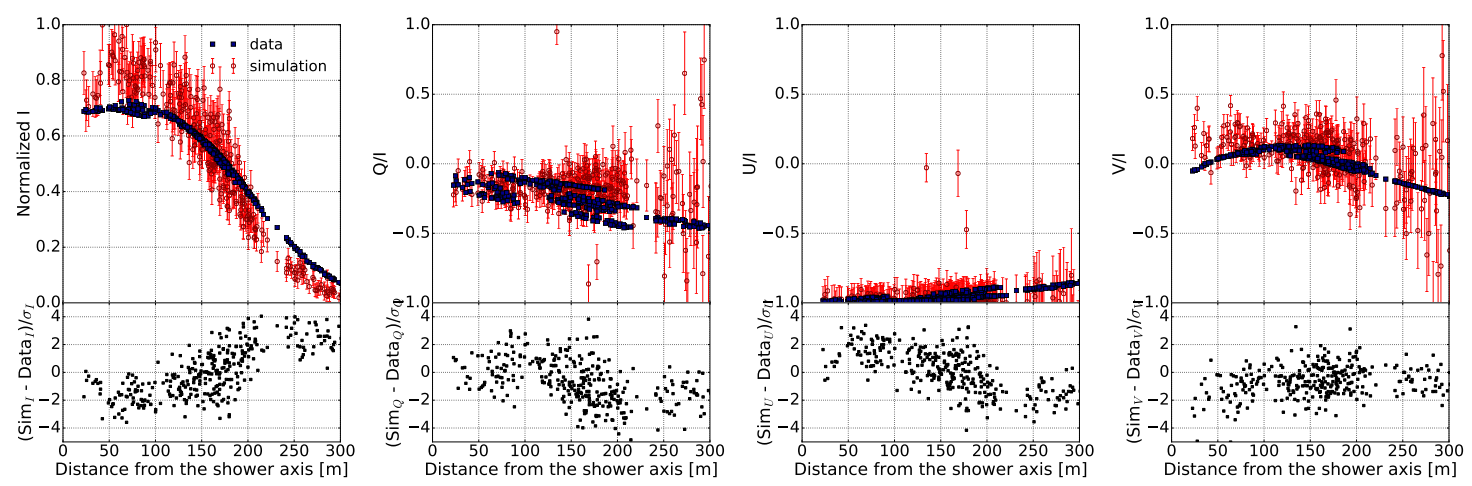

Figure 1: The results for normalized Stokes parameters (filled blue dots) calculated with CoREAS, using the field configuration given in Table 1 are compared to LOFAR data (open red circles) for event A. Bottom panel shows the difference between calculation and data normalized by $\sigma$, the one standard deviation error.
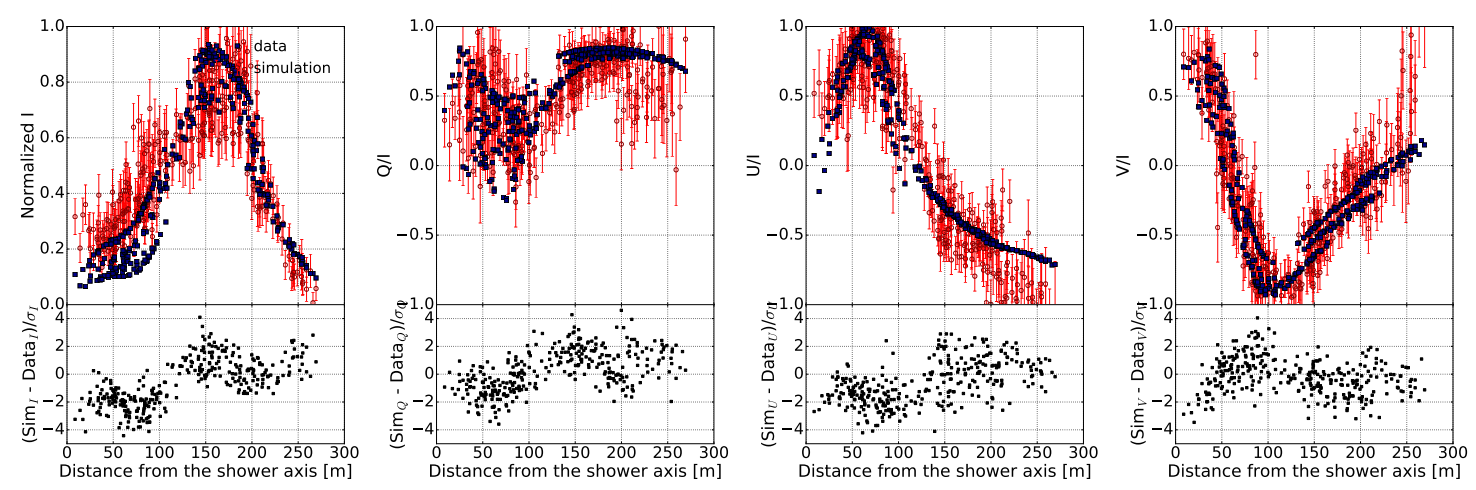

Figure 2: Same as Fig. 1 for event B.

\section{Extracting atmospheric electric fields}

From the values given in Table 1 one can see that the structure of the atmospheric electric field can be rather complicated. On the basis of the general charging mechanism in clouds one expects around the freezing level a positively charged layer, at $-10^{\circ} \mathrm{C}$ the main negative layer and near the top of the cloud another positive layer [9]. Based on this we assume a three-layered structure. The problem we are now facing is that for a measured radio footprint we have to search for the nine parameters that define such a three-layered configuration. This is too many parameters to deal with using CoREAS [15] because of running time and the stochastic nature of the calculations. To solve this problem we have developed a semi-analytic code MGMR3D [16, 17] that can calculate a complete radio footprint in just a few seconds. For this reason it can be used in a chi-square optimization. MGMR3D uses a simplified parametrization for the structure of the air shower and thus yields only an approximation to the full-scale calculation. To be sure about the configuration we thus perform a CoREAS [15] calculation where the atmospheric electric fields (found with MGMR3D) are implemented through the EFIELD option [18].

The first step in the procedure for constructing the atmospheric electric fields it thus to fit the measured cosmic-ray footprint using MGMR3D. This may require several attempts to ensure 


\begin{tabular}{|c|c|c|c|c|c|c|}
\hline Event & \multicolumn{3}{|c|}{ A } & \multicolumn{3}{|c|}{ B } \\
\hline Energy $(\mathrm{eV})$ & \multicolumn{3}{|c|}{$4.4 \times 10^{16}$} & \multicolumn{3}{|c|}{$3.9 \times 10^{16}$} \\
\hline Layer & $h$ & $E$ & $\alpha$ & $h$ & $E$ & $\alpha$ \\
\hline 1 & 9.1 & 57 & -63 & 5.8 & 30 & -29 \\
\hline 2 & 4.0 & 3 & 206 & 3.4 & 83 & 180 \\
\hline 3 & 1.2 & 4 & -20 & 1.7 & 13 & 30 \\
\hline$X_{\max }\left(\mathrm{g} / \mathrm{cm}^{2}\right)$ & \multicolumn{3}{|c|}{550} & \multicolumn{3}{|c|}{650} \\
\hline$X_{\max }(\mathrm{km})$ & \multicolumn{3}{|c|}{5.6} & \multicolumn{3}{|c|}{4.1} \\
\hline$\chi_{3 D}^{2}$ & \multicolumn{3}{|c|}{1.08} & \multicolumn{3}{|c|}{2.11} \\
\hline$\chi_{C}^{2}$ & \multicolumn{3}{|c|}{2.02} & \multicolumn{3}{|c|}{1.95} \\
\hline$f_{r}$ & \multicolumn{3}{|c|}{3} & \multicolumn{3}{|c|}{8} \\
\hline
\end{tabular}

Table 1: The values of the parameters describing the structure of the atmospheric electric field assuming three-layers. For each layer the top-height $h$ of the layer is given $[\mathrm{km}]$, the strength $E$ of the electric field $[\mathrm{kV} / \mathrm{m}]$, and the angle of the field with the $\mathbf{v} \times \mathbf{B}$ direction. Also given are the values for the chi-square for the MGMR3D calculation $\left(\chi_{3 D}^{2}\right)$ and that for CoREAS $\left(\chi_{C}^{2}\right)$ using the same field and almost the same $X_{\max }$. The normalization factor for the intensity of the radio signal is given by $f_{r}$.

that one is not stuck in a local minimum. It also turns out that the value of $X_{\max }$ is strongly correlated with the field configuration making it impossible to fit this in conjunction with the field configuration. We thus perform the fit for several fixed values for $X_{\max }$. In a second stage the obtained field configuration is used is a CoREAS calculation where also the measured scintillator signal is taken into account. The final selection is based on the agreement between CoREAS and the data as well as the absolute intensity of the radio emission (expressed by $f_{r}$ where $f_{r}=1$ implies good agreement and the energy of the shower is determined from the scintillator signal).

The thus obtained values are given in Table 1 and the quality of the CoREAS results can be judged from the value of $\chi_{C}^{2}$ in the table or even better from Fig. 1 and Fig. 2. One observes that in spite of rather complicated interferences between the emission from different heights the field configuration obtained from the MGMR3D calculations gives satisfactory results when used in CoREAS.

\subsection{E-Field Tomography}

The radio footprint is primarily sensitive to the field perpendicular to the shower axis. Thus, when two showers coming at different angles are measured within a short time span, one should be able to reconstruct the complete field. This we call E-Field Tomography. For this to work the basic assumption is that the fields does not change during the time between the showers as well as the the fields are the same along the tracks of the showers which do not coincide. It is difficult to give general scales for time and distances since this will depend on cloud size (typically $10 \mathrm{~km}$, but varies much), wind speeds. The tomography method also offers a test for the change in the field.

To perform tomography we consider two showers $i$ and $j$ with shower axes given by $\mathbf{v}_{i}$ and $\mathbf{v}_{j}$. For each of these the perpendicular components of the electric fields, $\mathbf{E}_{\perp i}$ and $\mathbf{E}_{\perp j}$, can be 
determined. Assuming that the complete field $\mathbf{E}$ is the same for the two showers, we may write

$$
\mathbf{E}_{\perp i}+E_{i \|} \mathbf{v}_{i}=\mathbf{E}=\mathbf{E}_{\perp j}+E_{j \|} \mathbf{v}_{j}
$$

where $E_{i \|}$ and $E_{j \|}$ are the parallel components of the fields. Taking the dot product of Eq. (4.1) with $e_{\mathbf{v}_{i} \times \mathbf{v}_{j}}, \mathbf{v}_{i}$ and $\mathbf{v}_{j}$ the following three equations are obtained,

$$
\mathbf{E}_{\perp i} \cdot\left(e_{\mathbf{v}_{i} \times \mathbf{v}_{j}}\right)=\mathbf{E}_{\perp j} \cdot\left(e_{\mathbf{v}_{i} \times \mathbf{v}_{j}}\right),
$$

and

$$
\begin{aligned}
E_{\| i} & =\frac{\mathbf{E}_{\perp j} \cdot \mathbf{v}_{i}+\left(\mathbf{v}_{i} \cdot \mathbf{v}_{j}\right)\left(\mathbf{E}_{\perp i} \cdot \mathbf{v}_{j}\right)}{1-\left(\mathbf{v}_{i} \cdot \mathbf{v}_{j}\right)^{2}} \\
E_{\| j} & =\frac{\mathbf{E}_{\perp i} \cdot \mathbf{v}_{j}+\left(\mathbf{v}_{i} \cdot \mathbf{v}_{j}\right)\left(\mathbf{E}_{\perp j} \cdot \mathbf{v}_{i}\right)}{1-\left(\mathbf{v}_{i} \cdot \mathbf{v}_{j}\right)^{2}} .
\end{aligned}
$$

Eq. (4.2) can be considered as a consistency check i.e. that both measurements yield the same component of the field in the $e_{\mathbf{v}_{i} \times \mathbf{v}_{j}}$-direction which is perpendicular to both showers. Eq. (4.3) allows the construction of the as yet missing parallel component of the field. This procedure can be applied to each layer separately.

\begin{tabular}{|c||c|c||c|c|}
\hline event A - event B & $\mathbf{E}_{A} \cdot\left(e_{\mathbf{v}_{A} \times \mathbf{v}_{B}}\right)$ & $\mathbf{E}_{B} \cdot\left(e_{\mathbf{v}_{A} \times \mathbf{v}_{B}}\right)$ & $\left(\mathbf{E}_{\perp A}+E_{\| A}\right)_{z}$ & $\left(\mathbf{E}_{\perp B}+E_{\| B}\right)_{z}$ \\
\hline \hline Bottom - Bottom & 4 & -9 & -15 & -15 \\
\hline Middle - Middle & -2 & 13 & 113 & 114 \\
\hline Top - Top & 43 & 10 & -94 & -95 \\
\hline
\end{tabular}

Table 2: Checking the consistency of electric fields extracted from events A and B (see Eq. (4.2)). The quoted values are in $[\mathrm{kV} / \mathrm{m}]$. The parallel components of the fields have been determined using Eq. (4.3) when reconstructing the vertical, $z$, component of the field.

In Table 2 we show the results when tomography is applied to events A and B. One sees that the consistency condition is obeyed to a reasonable extent. The table also gives the extracted vertical component of the field. This shows that the layer at an height of 4.0 or $3.4 \mathrm{~km}$ is strongly negatively charges. This is also close to the height of the $-10^{\circ}$-isotherm as expected. The $0^{\circ}$-isotherm lies at $2.5 \mathrm{~km}$ at the time of this event which is a bit higher than the height of the lower positive charge layer which we determine from these data. A more extensive discussion of these events will appear in Ref. [19]

\section{Summary}

We argue that atmospheric electric fields may have a large effect on the radio footprint of cosmic-ray air showers. We have shown the results for a measurement during an active thunderstorm, however strong electric fields have also been observed for heavy rain clouds.

It is shown that from the measured footprint it is possible to reconstruct the component of the electric field that is perpendicular to the shower. A semi-analytic code has been developed to be able to do so efficiently. Tomography can be applied when multiple events are measured within 
a short time-span. This allows for the reconstruction of the complete field and thus the charge structure in the cloud.

It should be noted that at GRAPES-3 muon telescope located in Ooty, India, strong variations in muons have been measured that have been used to determine potential differences in thunderclouds of $1.3 \mathrm{GV}$ [20]. Also at the Pierre Auger Observatory ring-like structures have been observed in the surface detectors with diameters of the order of a few $\mathrm{km}$ that can be explained as due to strong atmospheric electric fields of the order of $500 \mathrm{kV} / \mathrm{m}$ extending over a distance of a kilometer [21].

\section{Acknowledgments}

The LOFAR cosmic ray key science project acknowledges funding from an Advanced Grant of the European Research Council (FP/2007-2013) / ERC Grant Agreement n. 227610. The project has also received funding from the European Research Council (ERC) under the European Union's Horizon 2020 research and innovation programme (grant agreement No 640130). We furthermore acknowledge financial support from FOM, (FOM-project 12PR304) and NWO (VENI grant 639041-130). AN is supported by the DFG (research fellowship NE 2031/2-1).

LOFAR, the Low Frequency Array designed and constructed by ASTRON, has facilities in several countries, that are owned by various parties (each with their own funding sources), and that are collectively operated by the International LOFAR Telescope foundation under a joint scientific policy.

\section{References}

[1] Klaus Werner and Olaf Scholten, "Macroscopic Treatment of Radio Emission from Cosmic Ray Air Showers based on Shower Simulations", Astropart. Phys. 29, 393-411 (2008), arXiv:0712.2517.

[2] Olaf Scholten, Krijn D. de Vries, Klaus Werner, "What the radio signal tells about the cosmic-ray air shower", EPJ Web of Conf. 53, 08005 (2013), arXiv:1207.1874.

[3] T. Huege, "Radio detection of cosmic ray air showers in the digital era", Phys. Rep. 620, 1 (2016), arXiv:1601.07426.

[4] K. D. de Vries, et al., "Coherent Cherenkov Radiation from Cosmic-Ray-Induced Air Showers", Phys. Rev. Lett. 107, 061101 (2011).

[5] K. Werner, K.D. De Vries, O. Scholten, "A Realistic Treatment of Geomagnetic Cherenkov Radiation from Cosmic Ray Air Showers", Astropart. Phys. 37, 5 (2012), arXiv:1201.4471.

[6] Buitink, S., et al., "Method for high precision reconstruction of air shower X max using two-dimensional radio intensity profiles", Phys. Rev. D 90, 082003 (2014).

[7] S. Buitink, et al., "A large light-mass component of cosmic rays at $10^{17}-10^{17.5}$ electronvolts from radio observations", Nature 531, 70 (2016), corrigendum 537, 572 (2016); arXiv:1603.01594.

[8] G. Trinh, O. Scholten, et al., "Influence of Atmospheric Electric Fields on the Radio Emission from Extensive Air Showers.", Phys. Rev. D 93, 023003 (2016), arXiv:1511.03045.

[9] W. Deierling and W.A. Petersen, "Total lightning activity as an indicator of updraft characteristics", J. Geoph. Res.: Atmosph. 113(D16), D16210 (2008). 
[10] P. Schellart et al., "Probing Atmospheric Electric Fields in Thunderstorms through Radio Emission from Cosmic-Ray-Induced Air Showers", Phys. Rev. Lett. 114, 165001 (2015), arXiv:1504.05742.

[11] T.N.G.Trinh, et al., "Thunderstorm electric fields probed by extensive air showers through their polarized radio emission", Phys. Rev. D 95, 083004 (2017), arXiv:1703.06008.

[12] Gia Trinh et al., "Determining atmospheric electric fields through radio emission from air showers", Contribution to ARENA-2018.

[13] O. Scholten, et al., "Measurement of the circular polarization in radio emission from extensive air showers confirms emission mechanisms”, Phys. Rev. D 94, 103010 (2016), arXiv:1611.00758.

[14] M. P. van Haarlem et al., "LOFAR: The LOw-Frequency ARray”, Astron. \& Astrophys. 556, A2 (2013); arXiv:1305.3550.

[15] T. Huege, M. Ludwig, and C. James, "Simulating radio emission from air showers with CoREAS", AIP Conf. Proc. 1535, 128 (2012); arXiv:1301.2132.

[16] O. Scholten, T. N. G. Trinh, K. D. de Vries, and B. M. Hare (2018), "Analytic calculation of radio emission from parametrized extensive air showers: A tool to extract shower parameters", Phys. Rev. D 97, 023005 (2018).

[17] O. Scholten et al., "MGMR3D, a semi-analytic code for the obtaining the radio footprint from the shower currents", Contribution to ARENA-2018.

[18] S. Buitink, et al., "Monte Carlo simulations of air showers in atmospheric electric fields", Astropart. Phys. 33, 1 (2010).

[19] Gia Trinh, et al., "Atmospheric electric fields measured by LOFAR", to be published.

[20] B. Hariharan, et al., "Measurement of the Electrical Properties of a Thundercloud Through Muon Imaging by the GRAPES-3 Experiment", Phys. Rev. Lett. 122, 105101 (2019).

[21] Roberta Colalillo, et al., "SD rings as seen at the Pierre Auger Observatory: interpretation", These proceedings. 